\title{
Fast and ultrasensitive method for quantitating prion infectivity titre
}

\author{
Natallia Makarava1,2, Regina Savtchenko ${ }^{1,2}$, Irina Alexeeva ${ }^{3}$, Robert G. Rohwer ${ }^{3,4}$ \& Ilia V. Baskakov ${ }^{1,2}$
}

Bioassay by end-point dilution has been used for decades for routine determination of prion infectivity titre. Here we show that the new protein misfolding cyclic amplification with beads (PMCAb) technique can be used to estimate titres of the infection-specific forms of the prion protein with a higher level of precision and in 3-6 days as opposed to 2 years, when compared with the bioassay. For two hamster strains, $263 \mathrm{~K}$ and SSLOW, the median reactive doses determined by PCMAb (PMCA $b_{50}$ ) were found to be $10^{12.8}$ and $10^{12.2}$ per gram of brain tissue, which are 160- and 4,000-fold higher than the corresponding median infectious dose (ID50) values measured by bioassay. The $10^{2}$ - to $10^{3}$-fold differences between $I D_{50}$ and PMCA $b_{50}$ values could be due to a large excess of PMCAb-reactive prion protein seeds with little or no infectivity. Alternatively, the differences between $\mathrm{ID}_{50}$ and PMCAb 50 could be due to higher rate of clearance of infection-specific prion protein seeds in animals versus PMCAb reactions. A well-calibrated PMCAb reaction can be an efficient and cost-effective method for the estimation of infection-specific prion protein titre.

\footnotetext{
${ }^{1}$ Center for Biomedical Engineering and Technology, University of Maryland, 725 W. Lombard Street, Baltimore 21201, USA. ${ }^{2}$ Department of Anatomy and Neurobiology, University of Maryland School of Medicine, Baltimore 21201, USA. ${ }^{3}$ Medical Research Service, Veterans Affairs Maryland Health Care System, Baltimore 21201, USA. ${ }^{4}$ Department of Neurology, University of Maryland School of Medicine, Baltimore 21201, USA. Correspondence and requests for materials should be addressed to I.V.B. (email: Baskakov@umaryland.edu).
} 
A pathognomonic hallmark of the prion diseases is the accumulation of the misfolded isoform of the prion protein $\left(\mathrm{PrP}^{\mathrm{Sc}}\right)$. The traditional method for obtaining a quantitative estimate of prion infectivity is end-point dilution titration in animals. A suspension of the tissue or fluid of interest is diluted in tenfold serial steps, and then each dilution is inoculated into a group of animals. A dilution at which only a fraction of the inoculated animals develops clinical signs of disease or shows positive evidence of $\mathrm{PrP}^{\mathrm{Sc}}$ on immunoassay is called a 'limiting dilution'. At limiting dilution there are only one or a few infectious doses per inoculation volume. End-point dilution titres are typically expressed as the median infective dose $\left(\mathrm{ID}_{50}\right)$ : the reciprocal of the dilution required to infect only $50 \%$ of the animals inoculated as determined by interpolation or other statistical methods. While the end-point bioassay has been the principal method for determining prion infectivity, the assay is extremely long, expensive and laborious. Moreover, the bioassay works optimally only for prion strains with incubation times well within the lifespan of the host.

Alternatives to end-point titration are biochemical or immunochemical assays that assess either the presence, mass or concentration of $\mathrm{PrP}^{\mathrm{Sc}}$ (refs 1-4). However, establishing accurate quantitative relationships between $\mathrm{PrP}^{\mathrm{Sc}}$ concentration and prion titre has proven to be difficult because of the size heterogeneity of prion particles and uncertainty over whether all prion particles are equally infectious ${ }^{5-7}$. Moreover, the size distribution and physical properties of prion particles appear to vary with agent strain and host species ${ }^{6}$. In 2003, Weissmann and co-workers ${ }^{8}$ introduced a scrapie cell assay that quantitatively estimates prion infectivity titres within a much shorter time frame than animal bioassay. The scrapie cell assay was shown to be capable of detecting prions in a dilution as low as $10^{10}$-fold of scrapieinfected brain material ${ }^{9}$. Moreover, in recent studies, the scrapie cell assay was adapted for detecting prions from various species ${ }^{10}$.

Protein misfolding cyclic amplification (PMCA) propagates $\mathrm{PrP}^{\mathrm{Sc}}$ and infectivity in vitro ${ }^{11-14}$. The sensitivity of the PMCA reaction to detect prion particles exceeds that of the bioassay ${ }^{15,16}$. While PMCA has been proven for detecting and amplifying prions from a broad range of species including human, cow, sheep, cervids, mouse, hamster and others ${ }^{16-26}$, highly robust PMCA amplification has been limited to mostly rodent and rodent-adapted strains. Nevertheless, the efficiency of prion replication in PMCA was shown to mimic cross-species transmission barriers ${ }^{27-29}$ or genetic susceptibility of sheep to scrapie that occurs due to prion protein polymorphisms ${ }^{19}$. The improvements in the PMCA assay found in PMCA with beads (PMCAb) have resulted in a much faster, more robust, sensitive and cost-efficient way of measuring $\mathrm{PrPSc}^{\mathrm{Sc}}$ compared with either PMCA or bioassay ${ }^{29,30}$.

To illustrate the advantages of PMCAb-based end-point titration, we assessed the relative concentrations of $\mathrm{PrP}^{\mathrm{Sc}}$ in brain material of two rodent strains, $263 \mathrm{~K}$ and SSLOW, which display very short or very long incubation times to symptomatic disease, respectively ${ }^{31}$. We show that using the PMCAb format $\mathrm{PrP}^{\mathrm{Sc}}$ titration can be performed in only a few days. We also observed strain-specific differences in the specific infectivity relative to PMCAb-based $\mathrm{PrP}^{\mathrm{Sc}}$ titre ranging from hundreds to thousands of $\mathrm{PMCAb}$-active particles per intracranial infectious dose 50 (ic $\mathrm{ID}_{50}$ ).

\section{Results}

End-point dilution titration using PMCAb. The PMCAb amplification was initiated by 'seeding' a substrate of uninfected brain homogenate $(\mathrm{BH})$ with $\mathrm{PrP}^{\mathrm{Sc}}$. To determine the concentration of PMCAb seeds, $10 \%$ BHs prepared from $263 \mathrm{~K}$ - or SSLOWinfected animals were diluted in tenfold serial steps, then aliquots from each dilution were used to seed serial PMCAb reactions. Up to ten independent serial PMCAb reactions were conducted for each dilution for each strain. Each PMCAb round consisted of 48 cycles, 30 min each. Three or six serial PMCAb rounds were sufficient a

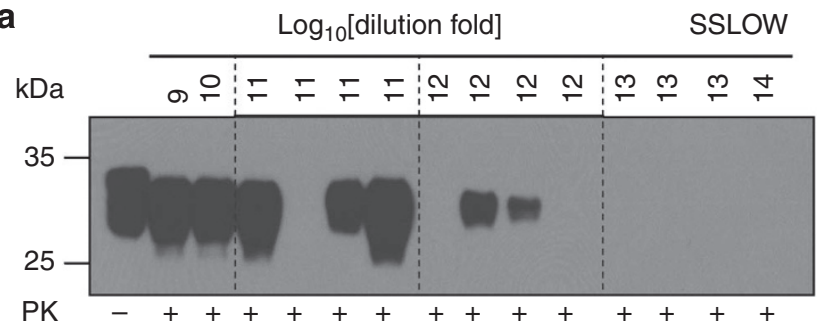

b
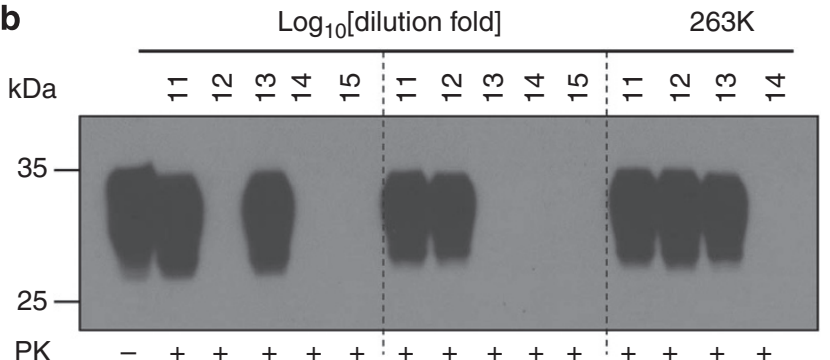

c
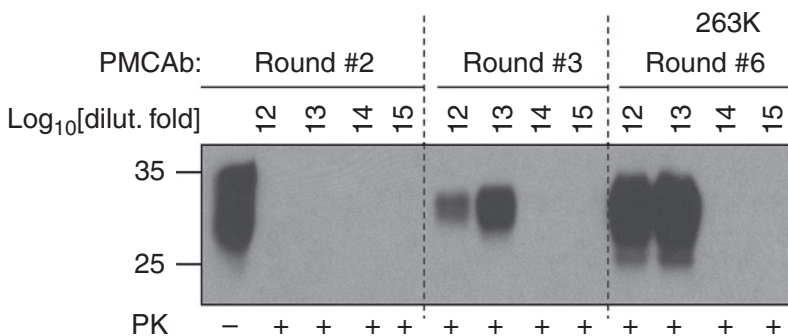

Figure 1 | End-point sPMCAb titration. 10\% scrapie brain material from SSLOW- (a) or $263 \mathrm{~K}$-infected animals $(\mathbf{b}, \mathbf{c})$ were diluted in tenfold serial steps, then aliquots from each dilution were used to seed serial PMCAb reactions. Example of a western blot of serial PMCAb (sPMCAb) products amplified for six rounds is shown in plots $\mathbf{a}$ and $\mathbf{b}$. Panel $\mathbf{c}$ shows products of PMCAb reactions seeded with four dilutions and amplified for 2,3 or 6 serial rounds. The dilutions shown for PMCAb reactions were normalized per gram of brain tissue, to correct for differences in assay volumes used for PMCAb $(100 \mu \mathrm{l})$ and bioassay $(50 \mu \mathrm{l})$.

for amplification of even the highest dilutions of $263 \mathrm{~K}$ or SSLOW, respectively (Fig. 1a,b), to the level detectible by western blot. An increase in the number of PMCAb rounds did not increase the percentage of positive reactions for the most highly diluted samples illustrating that the limiting dilution was reached (Fig. 1c).

In our experience, PMCAb displays a high level of selectivity for infection-specific forms of PrP. To date, we have detected no false-positive amplifications in the routine control reactions that are included with every PMCAb titration experiment. These controls consist of six serial rounds of 48 cycles of PMCAb of unseeded substrate only. Other controls include six rounds of serial PMCAb of $10 \%$ normal BHs from 700-day-old hamsters (Fig. 2), and PMCAb amplifications of hamster substrate seeded with full-length recombinant hamster PrP amyloid fibrils prepared under a variety of previously described experimental conditions and protocols ${ }^{32-35}$. No positive signals were detected in any of the controls (Fig. 2).

End-point dilution titration in animals. In parallel to PMCAb titration, the infectivity titres of brains from $263 \mathrm{~K}$ - and SSLOW-infected animals were measured using end-point dilution bioassay. Animals were considered infected if they develop symptomatic disease or if their brains contained $\operatorname{PrP}^{\mathrm{Sc}}$, as judged by western blot even without symptomatic disease. The fractions of animals infected at each dilution are presented in Table 1. 


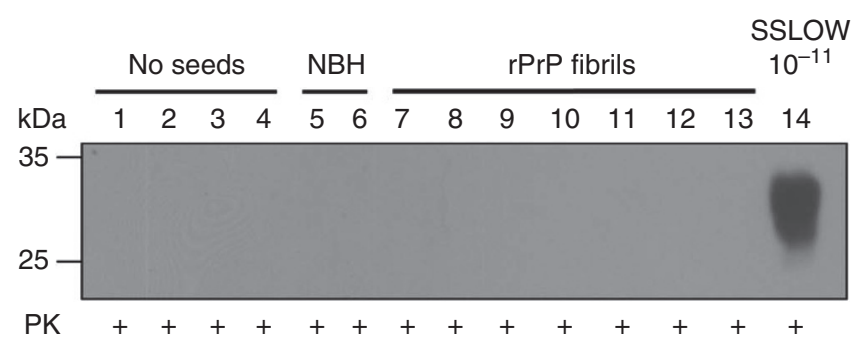

Figure 2 | Testing the specificity of PrPSc detection in sPMCAb. SPMCAb reactions were seeded with $10 \%$ NBH from 700-day-old Syrian hamsters (lanes 5 and 6), recombinant $\operatorname{PrP}(\mathrm{rPrP}$ ) amyloid fibrils type I not subjected to annealing (lane 7); fibrils type I subjected to annealing in Triton (lane 8), BSA (lane 9) or NBH (lanes 10, 11 and 12); with rPrP amyloid fibrils type II (lane 13); or 1011-fold diluted SSLOW brain material (lane14). Four nonseeded reactions are shown in lanes 1, 2, 3 and 4 . Six rounds of sPMCAb were conducted for each seeded condition and non-seeded reaction. The final concentration of rPrP amyloid fibrils in serial PMCAb reactions was $10 \mu \mathrm{g} \mathrm{ml}^{-1}$. rPrP fibrils were prepared using Syrian Hamster full-length $\mathrm{rPrP}$; fibrils type I were prepared in $2 \mathrm{M}$ guanidine hydrochloride $(\mathrm{GdnHCl})$ as described by Bocharova et al. ${ }^{32}$; fibrils type II were prepared in $0.5 \mathrm{M}$ $\mathrm{GdnHCl}$ as described by Sun et al. ${ }^{34} \mathrm{rPrP}$ fibrils type I were subjected to annealing in $1 \%$ Tritorn $\mathrm{X}-100,5 \mathrm{mg} \mathrm{ml}^{-1} \mathrm{BSA}$ or $5 \% \mathrm{NBH}$ as described by Bocharova et al. ${ }^{33}$ and Makarava et al. ${ }^{31}$. After six sPMCAb rounds, all samples were treated with PK as described in Methods; western blots were stained with 3F4. To rigorously test the specificity of sPMCAb for detecting $\mathrm{PrPS}^{\mathrm{Sc}}$, multiple experiments were performed using four Misonix S-4000 sonicators over a 2-year period. Several independently prepared stocks of rPrP amyloid fibrils of each type were produced using rPrP from several independent purifications. All results on sPMCAb seeding by $r P r P$ fibrils were negative, confirming that $\mathrm{SPMCAb}$ is highly specific for $\mathrm{Pr} P \mathrm{Sc}$.

Table 1 | Summary of end-point titration of $263 \mathrm{~K}$ and SSLOW.

Dilution of brain material Number of animals infected ${ }^{\mathrm{a}}$ /total number 263K SSLOW

$10^{1} \quad 8 / 8$

$10^{2}-8 / 8$

$10^{3}-8 / 8$

$10^{4}$

$\begin{array}{lll}10^{6} & 12 / 12 & 8 / 8 \\ 10^{7} & 11 / 11\end{array}$

$10^{7} \quad 11 / 11 \quad 5 / 8$

$10^{8} \quad 55 / 59 \quad 2 / 8$

$10^{9} \quad 107 / 153 \quad 0 / 8$

$10^{10} \quad 2 / 12 \quad 0 / 6$

$10^{11} \quad 0 / 12 \quad 0 / 8$

${ }^{a}$ Animals were considered infected if they were symptomatic or positive for $\mathrm{Pr} P \mathrm{Sc}$ by western blotting.

Analysis of prion titre. For each dilution, the fraction of animals infected or the fraction of PMCAb reactions with a positive signal on western blot was plotted against the logarithm of dilution for both 263K and SSLOW (Fig. 3). At limiting dilution, $\mathrm{PrP}^{\mathrm{Sc}}$ particles must necessarily assort randomly into the reaction aliquots, and their distribution is described by the Poisson equation. While there was a good fit to the Poisson equation by the animal infectivity data for both $263 \mathrm{~K}$ and SSLOW, the PMCAb titration curves for both strains showed a more gradual slope than predicted by the Poisson equation (Fig. 3a). This is consistent with increasing reaction efficiency at higher dilutions. One possibility is that dilution results in a concentration-dependent dissociation of aggregates, thereby releasing and increasing the concentration of PMCAb reactive centres.
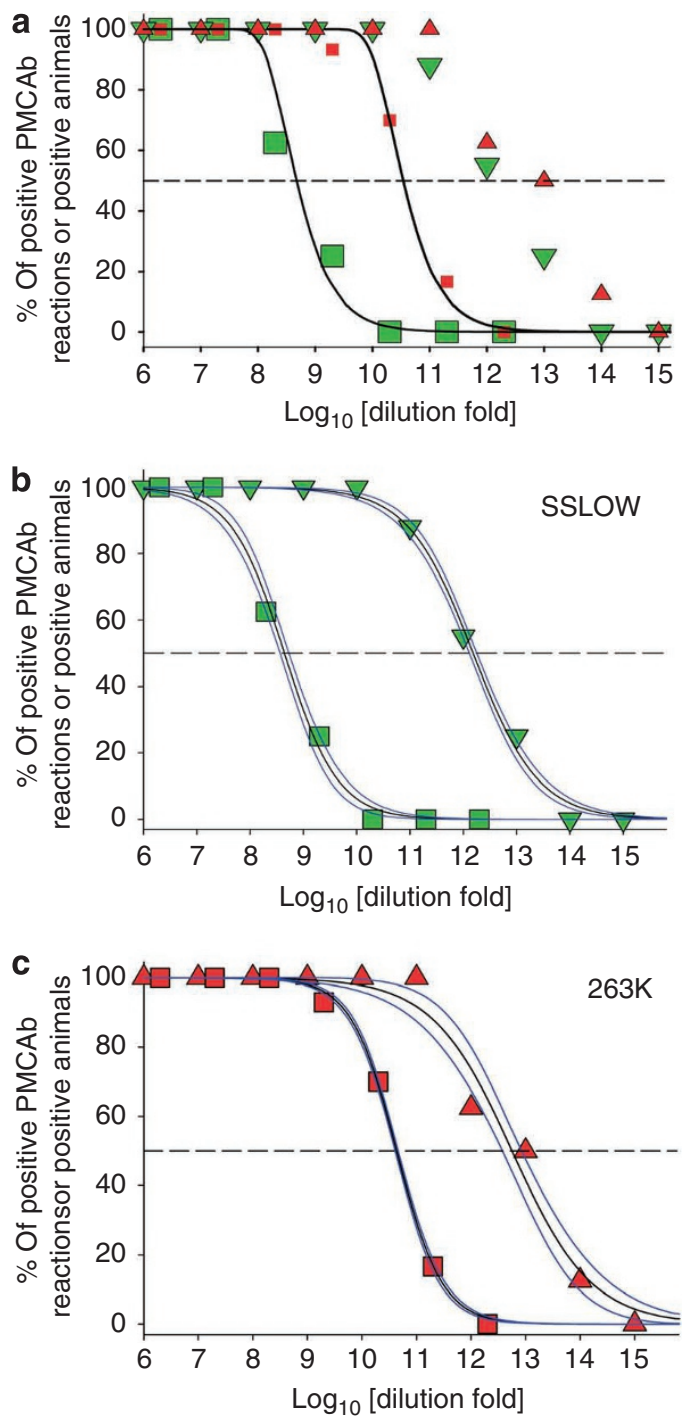

Figure 3 | Correlation of prion infectivity titre by end-point titration bioassay with PMCAb activity. Brain homogenate materials from Syrian Hamsters infected with SSLOW (large green symbols) or 263K (small red symbols) were subjected to ten-fold serial dilution, then each dilution was analysed by animal bioassay or serial PMCAb. Percentage of animals infected (squares) or giving positive PMCAb reactions (triangles) is plotted as a function of dilution. (a) Analysis of the data using the Poisson function, where the solid curves represent the results of nonlinear least-square best fit. Analysis of the data for SSLOW (b) or 263K (c) using a sigmoidal function. The solid curves represent the results of a nonlinear least-squares best fit and the blue curves represent $95 \%$ confidence intervals. ID 50 and PMCA $b_{50}$ values were calculated from the results of fitting.

Alternatively, dilution might diminish the effects of an inhibitor. As the effect is at very high dilutions, the inhibitor alternative seems less likely.

An arbitrary sigmoidal function and nonlinear regression analysis were used to calculate $\mathrm{ID}_{50}$ or $\mathrm{PMCAb}_{50}$ values from bioassayor PMCAb-based end-point curves, respectively (Fig. 3b,c, Table 2). (Note that the Poisson equation is a special instance of the more generalized sigmoidal function used.) The infectivity titres determined in this way were almost identical to those determined by the Poisson equation or by more traditional application of the Reed and Meunch and Spearman and Karber methods (Table 2). Analogous to the bioassay, a PMCAb $\mathrm{P}_{50}$ is the reciprocal of the concentration at which only $50 \%$ of the PMCAb reactions were positive. As judged 
Table 2 | ID 50 values measured by end-point bioassay or PMCAb.

\begin{tabular}{|c|c|c|c|c|}
\hline & \multicolumn{3}{|c|}{ Assay $_{50}$ units per gram of tissue } & \multirow{2}{*}{$\frac{\text { Ratio }}{\text { bPMCA }_{50} /^{\text {b }} \text { ID }_{50}}$} \\
\hline & Bioassay $^{\mathrm{a}} \mathrm{ID}_{50}$ & ${\text { Bioassayb } \text { ID }_{50}}$ & PMCAb $^{\text {b }}$ PMCAb $_{50}$ & \\
\hline 263K & $10^{10.5}$ & $10^{10.6}$ & $10^{12.8}$ & 160 \\
\hline SSLOW & $10^{8.7}$ & $10^{8.6}$ & $10^{12.2}$ & 4000 \\
\hline
\end{tabular}

from $\mathrm{ID}_{50}$ and $\mathrm{PMCAb}_{50}$ values, PMCAb was more sensitive than bioassay by $\sim 4,000$-fold for SSLOW and $\sim 160$-fold in the case of 263K. Importantly, PMCAb titration was completed in a few days, whereas the bioassay required nearly 2 years.

\section{Discussion}

We show here that PMCAb, when well calibrated against bioassay, can be used to obtain an estimate of $\mathrm{PrP}^{\mathrm{Sc}}$ titre in only 3-6 days, $\sim 100$ times faster than the bioassay. At the same time, PMCAb offers ethical advantages of reducing the need for animal use. The precision of the measurements in PMCAb is limited only by the number of replicates performed. The new PMCAb-based assay can be used as a fast, efficient and ultrasensitive method for determining $\mathrm{PrP}^{\mathrm{Sc}}$ titre and is uniquely beneficial for samples that have extremely low levels of infectivity and for determining infectivity concentrations for prion strains with long incubation times. On the other hand, while PMCA has been used for amplification of $\mathrm{PrP}^{\mathrm{Sc}}$ from a wide range of species $^{16,18-25}$, the most robust amplification was achieved, so far, for a limited number of rodent or rodent-adapted strains. Further technical improvements are needed for PMCA to be used as a platform for quantitative estimates of $\mathrm{PrP}^{\mathrm{Sc}}$ from a broad range of species.

In recent studies, Soto and co-workers ${ }^{16}$ estimated the amount of $\mathrm{PrP}^{\mathrm{Sc}}$ based on the number of PMCA rounds necessary to amplify prions to a detectible level. While PMCAb and PMCA methods have similar sensitivities for detection of $263 \mathrm{~K}$, PMCA requires 18 days to reach sensitivity comparable with that achieved by PMCAb in 3 days. Furthermore, PMCA did not work well for amplification of SSLOW $\mathrm{PrP}^{\mathrm{Sc}}$ (ref. 29). PMCAb is also far less sensitive to inhibitors and small variations in sonication conditions that have plagued conventional PMCA.

It is of interest to consider the molecular relationship of PMCAb titre to prion infectivity. A PMCAb ${ }_{50}$ titre represents the number of $\mathrm{PMCAb}$-active, $\mathrm{PrP}^{\mathrm{Sc}}$-particles capable of initiating PMCAb amplification per gram of material sampled. Both PMCAb amplification products and $\mathrm{PrP}^{\mathrm{Sc}}$ are heterogeneous in at least size, and perhaps structure, and exactly which structures represent infectivity is not yet clear. Furthermore, contradictory data exist regarding the specific infectivity of PMCA-generated versus that of brain-derived $\operatorname{PrP}^{\mathrm{Sc}}$ (refs 36,37).

Close comparison of the two sets of data, obtained from the end-point dilution titration on animals and PMCAb, can be used to establish a quantitative relationship. The ratio of $\mathrm{PMCAb_{50 }} / \mathrm{ID}_{50}$ is the number of PMCAb-active particles that corresponds to one prion infectious dose or unit. The $\mathrm{PMCAb}_{50} / \mathrm{ID}_{50}$ ratio was 160 and 4,000 particles per one infectious dose for $263 \mathrm{~K}$ and SSLOW, respectively (Table 2 ). There are several ways to explain the $10^{2}$ to $10^{3}$-fold excess in the number of PMCAb-active particles over the number of the infectious doses. The large $P M C A b_{50} / I_{50}$ ratio could be interpreted as only a few or one out of each 160 or 4,000 $\mathrm{PrP}^{\mathrm{Sc}}$ particles being truly infectious, whereas the majority of particles while detectible and amplifiable by PMCAb have little or no infectivity. Consistent with this interpretation, recent studies reported a dissociation between PMCA seeding ability and biological infectivity for $\mathrm{PrPSc}^{\mathrm{Sc}}$ seeds produced from $\mathrm{PrP}$ constructs with deletions of polybasic domains ${ }^{38}$. Furthermore, the studies by
Klingeborn et al. ${ }^{36}$ showed that $\mathrm{PrP}^{\mathrm{Sc}}$ generated in PMCA had considerably lower infectivity titre than that of brain-derived $\mathrm{PrP}^{\mathrm{Sc}}$ (ref. 36). This work suggested that two competitive PMCA pathways that amplify infectious $\mathrm{PrP}^{\mathrm{Sc}}$ and non-infectious particles exist. In contrast, the work by Shikiya and Bartz ${ }^{37}$ reported that PMCAgenerated $\mathrm{PrP}^{\mathrm{Sc}}$ had a titre similar to that of brain-derived $\mathrm{Pr} \mathrm{P}^{\mathrm{Sc}}$. Further studies are needed to clarify these contradictory results.

Alternatively, the differences between $\mathrm{ID}_{50}$ and $\mathrm{PMCAb}_{50}$ values might reflect substantial differences in obstacles to successfully initiate prion infection in an animal versus PMCAb reaction. It has been reported that prions inoculated into animals are subject to intensive proteolytic degradation and clearance ${ }^{39}$. In contrast, the PMCAb amplification is conducted in the presence of protease inhibitors to prevent degradation of the normal prion protein substrate for conversion. It is conceivable that this environment also preserves a greater fraction of infectious PMCAb-active particles, thereby accounting for the $\sim 10^{2}$ - to $10^{3}$-fold greater sensitivity of the PMCAb assay.

Strain 263K (short incubation, short clinical duration) and SSLOW (very long incubation and clinical duration ${ }^{31}$ ) represent the two extremes of prion hamster disease. Nevertheless, the concentration of PMCAb-reactive particles was similar in brains of animals infected with $263 \mathrm{~K}$ or SSLOW (Table 2). No PMCAb-reactive particles were found in brains from aged animals, confirming that these particles are disease-specific. In contrast to having similar PMCAb $\mathrm{b}_{50}$ values, the infectivity titre was $\sim 100$-fold lower for SSLOW than for $263 \mathrm{~K}$ (Table 2). The $\mathrm{PMCAb}_{50} / \mathrm{ID}_{50}$ ratio was 160 and 4,000 particles for $263 \mathrm{~K}$ and SSLOW, respectively (Table 2). If the hypothesis that $\mathrm{PMCAb}$ amplifies infectious and non-infectious prion protein seeds is correct, then $\mathrm{PMCAb}_{50} / \mathrm{ID}_{50}$ value reflects a strain-specific ratio of non-infectious, disease-associated PMCAb-reactive particles per infectious $\mathrm{PrP}^{\mathrm{Sc}}$ seeds. Alternatively, the difference in the $\mathrm{PMCAb}_{50} /$ $\mathrm{ID}_{50}$ ratio could also reflect strain-specific differences in the amount of PMCAb-active particles required to infect an animal. The difference in efficiency of infection between the two strains could be attributed to several factors. First, the differences could be due to the differences in strain-specific rates of $\mathrm{PrP}^{\mathrm{Sc}}$ clearance on inoculation. Second, the $\mathrm{PrP}^{\mathrm{Sc}}$ species that is the most reactive in PMCAb might be not the one that is the most toxic. In fact, previous studies pointed to an uncoupling of prion infectious titre and neurotoxicity ${ }^{40}$. While $\mathrm{PMCAb}$ presumably counts $\mathrm{PrP}^{\mathrm{Sc}}$ particles, the read-out parameter for bioassay is the presence of clinical or subclinical disease, which results from an accumulation of neurotoxic PrP species. Therefore, if infectious and neurotoxic PrP species are two different entities, the differences in $\mathrm{PMCAb}_{50} / \mathrm{ID}_{50}$ value could reflect a complex strainspecific relationship between a PMCAb-active $\operatorname{PrP}^{\mathrm{Sc}}$ species and a neurotoxic species.

The strain-specific difference in the $\mathrm{PMCAb}_{50} / \mathrm{ID}_{50}$ ratio was mainly due to the substantially lower ID $_{50}$ titre of SSLOW in comparison with that of $263 \mathrm{~K}$. We do not know whether we would have eventually seen an $\mathrm{ID}_{50}$ for SSLOW equivalent to that of $263 \mathrm{~K}$, if hamsters had longer lifespans in which to develop infections from low titre inocula. In this respect, PMCAb offers advantages over the bioassay for titering slow prion strains, as the read-out parameter in PMCAb is not limited by the lifespan of an animal. 
Further comparison of end-point titration data from bioassay and PMCAb will test whether $\mathrm{PMCAb}_{50} / \mathrm{ID}_{50}$ ratio represents an inherent strain-specific property. Fortunately, uncertainties as to the relationship of PMCAb-active particles to infectivity do not diminish the power or usefulness of PMCAb as a quantitative assay. $\mathrm{PMCAb}$ can be used as is, as a relative indicator of infectivity titre between two similar samples. With careful calibration and sample control, it can be used to estimate $\mathrm{PrP}^{\mathrm{Sc}}$ infectivity titre itself.

\section{Methods}

End-point titration bioassay. This study was carried out in strict accordance with the recommendations in the Guide for the Care and Use of Laboratory Animals of the National Institutes of Health. The protocol was approved by the Institutional Animal Care and Use Committee of the University of Maryland, Baltimore (assurance number: A32000-01; permit number: 0309001).

Ten percent scrapie $\mathrm{BH}$ was prepared in $\mathrm{PBS}, \mathrm{pH} 7.4$, by sonication and serially diluted up to $10^{11}$-fold in PBS, as previously described ${ }^{41}$. SSLOW-inoculated animals from the second passage of SSLOW ${ }^{31}$ were used for both bioassay and PMCAb. Before inoculation, samples were dispersed by $90 \mathrm{~s}$ of maximum power ultrasonication in PBS. Each hamster received 50- $\mu$ inoculum intracerebrally under general anaesthesia (2 $\mathrm{LPM} \mathrm{O}_{2} / 4 \mathrm{MAC}$ isoflurane). The inoculated animals were observed closely for up to 660 days post-inoculation or until they developed clinical signs of prion disease. For SSLOW-inoculated animals, the clinical signs were observed as early as $318 \pm 10$ days post-inoculation for the 10 -fold dilution of brain material or as late as $560 \pm 10$ days for the $10^{6}$-fold dilution of brain material. Only one out of eight animals inoculated with the $10^{7}$-fold dilution showed clinical signs, whereas no clinical signs were observed for dilutions $10^{8}$-fold and higher. Affected animals were euthanized and their disease status was confirmed by western blot analysis of their brains. At the end of the incubation (660 days post-inoculation) all remaining animals were euthanized, and all brains were assessed for the presence of $\mathrm{PrP}^{\mathrm{Sc}}$ by western blot. Animal brains that contained $\mathrm{PrP}^{\mathrm{Sc}}$ but had not yet developed symptomatic disease were considered infected.

End-point titration using PMCAb. Healthy hamsters were euthanized and immediately perfused with PBS, pH 7.4, supplemented with 5 mM EDTA. Brains were dissected, and $10 \% \mathrm{BH}$ (wt/vol) was prepared using ice-cold conversion buffer and glass/Teflon tissue grinders cooled on ice and attached to a constant torque homogenizer (Heidolph, RZR2020). The brains were ground at low speed until homogeneous, and then five additional strokes completed the homogenization. The composition of conversion buffer was as previously described ${ }^{11}: \mathrm{Ca}^{2+}$-free and $\mathrm{Mg}^{2+}$-free PBS, pH 7.4, supplemented with $0.15 \mathrm{M} \mathrm{NaCl}, 1.0 \%$ Triton and 1 tablet of Complete protease inhibitors cocktail (Roche, cat. no. 1836145) per $50 \mathrm{ml}$ of conversion buffer. The resulting $10 \%$ normal BH (NBH) in conversion buffer was used as the substrate in PMCA reactions. To prepare seeds, scrapie-infected brains were homogenized as for inoculation (above), and $100 \mu$ l aliquots were sonicated in MISONIX S-400 microplate (Misonix) horn for $30 \mathrm{~s}$ at $50 \%$ power before serial dilution from 10 - to $10^{14}$-fold in conversion buffer. Then, $10 \mu \mathrm{l}$ of each dilution were used to seed $90 \mu \mathrm{l}$ of $\mathrm{NBH}$ for PMCAb. Teflon beads $(2.38 \mathrm{~mm}$ diameter, McMaster-Carr, Los Angeles, CA, USA) were placed into the $0.2 \mathrm{ml}$ tubes first, and then NBH and seeds were added. Samples in $0.2 \mathrm{ml}$ thin-wall PCR tubes (Fisher, cat. no. 14230205) were placed in a floating rack inside a Misonix $\mathrm{S}-4000$ microplate cup horn filled with $350 \mathrm{ml}$ water. Two coils of rubber tubing attached to a circulating water bath were installed for maintaining $37^{\circ} \mathrm{C}$ inside the sonicator chamber. The standard sonication program consisted of $30 \mathrm{~s}$ sonication pulses delivered at $50 \%$ efficiency applied every 30 min during a $24-\mathrm{h}$ period. For each subsequent round of serial PMCAb, $10 \mu \mathrm{l}$ aliquotes from a previous round were used to seed the reactions in the next round. At limiting dilutions, up to ten independent serial PMCAb reactions were used for each dilution to accumulate statistics.

Data analysis. To correct for differences in assay volumes used for PMCAb $(100 \mu \mathrm{l})$ and bioassay $(50 \mu \mathrm{l})$, the fraction of positive PMCAb reactions or positive animals, presented in Fig. 3, were normalized per gram of brain tissue.

Analysis using Poisson equation. For the probability that a given aliquot received no active particle, $P(0)$, the Poisson equation reduces to $P(0)=\mathrm{e}^{-m}$, where $m$ is the concentration of the active particle at that dilution in particles per inoculation volume. The probability that there will be at least one active particle to initiate infection, $P(\geq 1)$, is $P(\geq 1)=1-\mathrm{e}^{-m}$. The infectivity data were fit to the expression:

$$
F=1-\exp \left(-\mathrm{a}^{*} x\right)
$$

where $F$ is fraction of infected animals, $x$ is dilution and $a$ is the fitted parameter equal to the undiluted concentration of infectivity. $\mathrm{ID}_{50}$ was calculated by solving the fitted equations for $F=0.5$ (infectivity), which reduce to the expression:

$$
\mathrm{ID}_{50}=-\ln (0.5) / \mathrm{a}
$$

Analysis using sigmoidal equation. $\mathrm{ID}_{50}$ and $\mathrm{PMCAb}_{50}$ values were determined by regression analysis in Sigma Plot using nonlinear least squares fitting of both sets of data to the sigmoidal equation:

$$
F=\left(100^{\star} \exp \left(-\left(A+B^{\star} x\right)\right)\right) /\left(1+\exp \left(-\left(A+B^{\star} x\right)\right)\right)
$$

where $F$ is percent of positive PMCAb reactions or infected animals, $x$ is logarithm of the dilution fold, $A$ and $B$ are two fitting parameters that define the position of a limiting dilution transition on the $x$ axis and the slope of the transition, respectively. $\mathrm{ID}_{50}$ and $\mathrm{PMCAb}_{50}$ were calculated according to the equation:

$$
\mathrm{PMCAb}_{50} \text { or } \mathrm{ID}_{50}=A / B
$$

Proteinase K assay. To analyse the PMCAb end-point titration reactions, $10 \mu \mathrm{l}$ of each sample was supplemented with $5 \mu \mathrm{l}$ SDS and $5 \mu \mathrm{l}$ proteinase $\mathrm{K}(\mathrm{PK})$, to a final concentration of SDS and PK of $0.25 \%$ and $50 \mu \mathrm{g} \mathrm{ml}^{-1}$, respectively, followed by incubation at $37^{\circ} \mathrm{C}$ for $1 \mathrm{~h}$. The digestion was terminated by addition of SDS sample buffer and boiling for $10 \mathrm{~min}$. Samples were loaded onto NuPAGE 12\% BisTris gels, transferred to polyvinylidene difluoride membrane, and stained with 3F4 antibody.

To analyse scrapie $\mathrm{BHs}$, an aliquot of $10 \% \mathrm{BH}$ was mixed with an equal volume of $4 \%$ sarcosyl in PBS, supplemented with $50 \mathrm{mM}$ Tris, $\mathrm{pH} 7.5$, and digested with $20 \mu \mathrm{g} \mathrm{ml}^{-1} \mathrm{PK}$ for $30 \mathrm{~min}$ at $37^{\circ} \mathrm{C}$ with 1,000 r.p.m. shaking (Eppendorf Thermomixer). The reaction was stopped by SDS sample buffer. Samples were boiled for $10 \mathrm{~min}$ and loaded onto NuPAGE $12 \%$ BisTris gels. After transfer to polyvinylidene difluoride membrane, PrP was detected with 3F4 antibody.

\section{References}

1. Wadsworth, J. D. et al. Tissues distribution of protease resistant prion protein in variant Creutzfeldt-Jakob disease using a highly sensitive immunobloting assay. Lancet 358, 171-180 (2001).

2. Safar, J. G. et al. Measuring prions causing bovine spongiform encephalopathy or chronic wasting disease by immunoassays and transgenic mice. Nat. Biotechnol. 20, 1147-1150 (2002).

3. Edgeworth, J. A. et al. Detection of prion infection in variant Creutzfeldt-Jakob disease: a blood-based assay. Lancet 377, 487-493 (2011).

4. Gregori, L. et al. A sensitive and quantitative assay for normal PrP in plasma. J. Virol Methods 149, 251-259 (2008).

5. Silveira, J. R. et al. The most infectious prion protein particles. Nature 437, 257-261 (2005)

6. Tixador, P. et al. The physical relationship between infectivity and prion protein aggregates is strain-dependent. PLOS Pathog. 6, e1000859 (2010).

7. Barron, R. M. et al. High titer of transmissible spongiform encephalopathy infectivity associated with extremely low levels of PrPSc in vitro. J. Biol. Chem. 35878-35886 (2007).

8. Klöhn, P. C., Stoltze, L., Flechsig, E., Enari, M. \& Weissmann, C. A quantitative, highly sensitive cell-based infectivity assay for mouse scrapie prions. Proc. Acad. Natl Sci. USA 100, 11666-11671 (2003).

9. Edgeworth, J. A., Jackson, G. S., Clarke, A. R., Weissmann, C. \& Collinge, J. Highly sensitive, quantitative cell-based assay for prions adsorbed to solid surfaces. Proc. Acad. Natl Sci. USA 106, 3479-3483 (2009).

10. Arellano-Anaya, Z. E. et al. A Simple, versatile and sensitive cell-based assay for prions from various species. Plos ONE 6, e20563 (2011).

11. Castilla, J., Saa, P., Hetz, C. \& Soto, C. In vitro generation of infectious scrapie prions. Cell 121, 195-206 (2005).

12. Saborio, G. P., Permanne, B. \& Soto, C. Sensitive detection of pathological prion protein by cyclic amplification of protein misfolding. Nature 411, 810-813 (2001).

13. Deleault, N. R., Harris, B. T., Rees, J. R. \& Supattapone, S. Formation of native prions from minimal components in vitro. Proc. Acad. Natl Sci. USA 104, 9741-9746 (2007).

14. Wang, F., Wang, X., Yuan, C.- G. \& Ma, J. Generating a prion bacterially expressed recombinant prion protein. Science 327, 1132-1135 (2010).

15. Saa, P., Castilla, J. \& Soto, C. Ultra-efficient replication of infectious prions by automated protein misfolding cyclic amplification. J. Biol. Chem. 281, 35245-35252 (2006).

16. Chen, B., Morales, R., Barria, M. A. \& Soto, C. Estimating prion concentration in fluids and tissues by quantitative PMCA. Nat. Methods 7, 519-520 (2010).

17. Deleault, N. R., Lucassen, R. W. \& Supattapone, S. RNA molecules stimulate prion protein conversion. Nature 425, 717-720 (2003).

18. Haley, N. J. et al. Detection of chronic wasting disease prions in salivary, urinary, and intestinal tissues of deer: potential mechanisms of prion shedding and transmission. J. Virol. 85, 6309-6318 (2011).

19. Bucalossi, C. et al. Assessment of the genetic susceptibility of sheep to scrapie by protein misfolding cyclic amplification and comparison with experimental scrapie transmission studies. J. Virol. 85, 8386-8392 (2011).

20. Yokoyama, T. et al. Heparin enhances the cell-protein misfolding cyclic amplification efficiency of variant Creutzfeldt-Jakob disease. Nerosci. Lett. 498, 119-123 (2011). 
21. Ayers, J. L. et al. The strain-encoded relationship between PrP replication, stability and processing in neurons is predictive of the incubation period of disease. PLOS Pathog. 7, e1001317 (2011).

22. Murayama, Y. et al. Sulfated dextrans enhance in vitro amplification of bovine spongiform encephalopathy $\operatorname{PrP}(\mathrm{Sc})$ and enable ultrasensitive detection of bovine $\operatorname{PrP}(\mathrm{Sc})$. Plos ONE 5, e13152 (2010).

23. Tattum, M. H., Jones, S., Pal, S., Collinge, J. \& Jackson, G. S. Discrimination between prion-infected and normal blood samples by protein misfolding cyclic amplification. Transfusion 50, 2619-2627 (2010).

24. Rubenstein, R. et al. Prion disease detection, PMCA kinetics, and IgG in urine from sheep naturally/experimentally infected with scrapie and deer with preclinical/clinical chronic wasting disease. J. Virol. 85, 9031-9038 (2011).

25. Nishina, K. et al. The stoichiometry of host PrPC glycoforms modulates the efficiency of PrPSc formation in vitro. Biochemistry 45, 14129-14139 (2006).

26. Gonzalez-Montalban, N., Makarava, N., Savtchenko, R. \& Baskakov, I. V. Relationship between conformational stability and amplification efficiency of prions. Biochemistry 50, 7933-7940 (2011)

27. Castilla, J. et al. Crossing the species barrier by PrPSc replication in vitro generates unique infectious prions. Cell 134, 757-768 (2008).

28. Green, K. M. et al. Accelerated high fidelity prion amplification within and across prion species barriers. PLOS Pathog. 4, e1000139 (2008).

29. Gonzalez-Montalban, N. et al. Highly efficient protein misfolding cyclic amplification. PLoS Pathog. 7, e1001277 (2011).

30. Pritzkow, S. et al. Quantitative detection and biological propagation of scrapie seeding activity in vitro facilitate use of prions as model pathogens for disinfection. Plos ONE 6, e20384 (2011).

31. Makarava, N. et al. Recombinant prion protein induces a new transmissible prion disease in wild type animals. Acta Neuropathol. 119, 177-187 (2010).

32. Bocharova, O. V., Breydo, L., Parfenov, A. S., Salnikov, V. V. \& Baskakov, I. V. In vitro conversion of full length mammalian prion protein produces amyloid form with physical property of PrPSc. J. Mol. Biol. 346, 645-659 (2005).

33. Bocharova, O. V. et al. Annealing PrP amyloid firbils at high temperature results in extension of a proteinase K resistant core. J. Biol. Chem. 281, 2373-2379 (2006).

34. Sun, Y. et al. Conformational stability of PrP amyloid firbils controls their smallest possible fragment size. J. Mol. Biol. 376, 1155-1167 (2008).
35. Makarava, N. \& Baskakov, I. V. The same primary structure of the prion protein yields two distinct self-propagating states. J. Biol. Chem. 283, 15988-15996 (2008).

36. Klingeborn, M., Race, B., Meade-White, K. D. \& Chesebro, B. Lower specific infectivity of protease-resistant prion protein generated in cell-free reactions. Proc. Acad. Natl Sci. USA 108, 1244-1253 (2011).

37. Shikiya, A. S. \& Bartz, J. C. In vitro generation of high titer prions. J. Virol. 85 , 13439-13442 (2011).

38. Miller, M. B., Geoghegan, J. C. \& Supattapone, S. Dissociation of infectivity from seeding ability in prions with alternate docking mechanism. PLoS Pathog 7, e1002128 (2011)

39. Safar, J. et al. Prion clearance in bigenic mice. J. Gen. Virol. 86, 2913-2923 (2005)

40. Sandberg, M. K., Al-Doujaily, H., Sharps, B., Clarke, A. R. \& Collinge, J. Prion propagation and toxicity in vitro occur in two distinct mechanistic phases. Nature 470, 540-542 (2011).

41. Gregori, L. et al. Reduction of transmissible spongiform encephalopathy infectivity from human red blood cells with prion protein affinity ligands. Transfusion 46, 1152-1161 (2006).

\section{Acknowledgements}

We thank Pamela Wright for editing the manuscript. This work was supported by NIH grant NS045585 to I.V.B., and VA Merit Award to R.G.R.

\section{Author contributions}

N.M., R.S. and I.A. carried out the work. N.M., R.G.R. and I.V.B. designed the study and analysed the data. I.V.B and R.G.R. wrote the manuscript. All authors commented on the final manuscript.

\section{Additional information}

Competing financial interests: The authors declare no competing financial interests.

Reprints and permission information is available online at http://npg.nature.com/ reprintsandpermissions/

How to cite this article: Makarava, N. et al. Fast and ultrasensitive method for quantitating prion infectivity titre. Nat. Commun. 3:741 doi: 10.1038/ncomms1730 (2012). 